\title{
Georges Ha Van (2008) Le pied diabétique, éditions Masson, Paris, 201 pages, ISBN 978-2-294-70257-0
}

\author{
S. Bendaya \\ (C) Springer-Verlag 2009
}

Ce livre préfacé par le Pr Grimaldi, chef de service de diabétologie du CHU de La Pitié-Salpêtrière, est un véritable plaidoyer pour une prise en charge multidisciplinaire structurée et programmée selon un algorithme bien déterminé au sein de centres de références. Il fait appel à une parfaite connaissance interdisciplinaire permettant de contrôler tous les facteurs de risques métaboliques, mécaniques, vasculaires, neurologiques et infectieux. Il offre des solutions émanant de la riche expérience de l'auteur, dont l'application permettra sans doute d'éviter bien des écueils liés à des taux d'amputations encore trop élevés. Ce livre traite d'un véritable problème de santé publique. Il analyse, dans un ordre chronologique, d'abord les causes de survenue d'une plaie du pied diabétique, grâce à l'étude des différents facteurs de risque. À cet égard, quatre principales causes sont analysées. L'élément vasculaire, par sa microangiopathie spécifique, l'artériosclérose avec la médiacalcose et l'athéromatose sont des facteurs aggravants. L'étude morphostatique, selon Georges $\mathrm{Ha}$ Van, est une étape incontournable dans l'analyse du risque mécanique, avec en particulier l'analyse du chaussage et de l'aspect trophique du pied, les orthèses plantaires, les systèmes de décharge et les chaussures thérapeutiques sur mesure font partie de l'arsenal thérapeutique. L'infection est classée en fonction de sa gravité en stades, et la neuropathie en général est source d'indolence et de complications longtemps méconnues, à l'origine soit de mal perforant plantaire, d'ostéoarthropathie neurogène ou de maladie de Charcot du pied. Des règles de prévention primaires et secondaires sont ensuite édictées. Dans un ordre logique, sont abordés secondairement les principes de prise en charge qui font appel à un examen méthodique vasculaire, neurologique trophique et mécanique. Ce dernier est documenté par des examens vasculaires incontournables comportant notamment la prise de pression de cheville, l'écho-doppler artériel des membres, la mesure de la pression transcutanée en oxygène et l'artériographie. Les prélèvements bactériologiques sont indispensables dans la perspective du traitement anti-infectieux. Vient ensuite le traitement de la plaie qui sera élaboré après décision médicochirurgicale, contrôle des facteurs métaboliques et après avoir réalisé au préalable un bilan vasculaire. En dehors du contrôle de l'infection, une grande part du traitement est basée sur la mise en décharge et la cicatrisation dirigée. La chirurgie doit être dans la mesure du possible conservatrice, associant en fonction des cas : revascularisation, débridement, détersion manuelle. L'amputation reste le dernier recours, son niveau sera guidé par l'état artériel. Une part importante de ce livre est réservée à l'éducation thérapeutique et ouvre des perspectives d'avenir sur l'importance de l'existence de centre de référence ou d'unité multidisciplinaire de podologie en diabétologie. Malgré la prise en charge récente et partielle des soins de pédicurie des patients à haut risque podologique, des progrès restent à accomplir dans le remboursement des actes d'éducation thérapeutique. Des efforts sont également à fournir dans la formation des médecins et des paramédicaux qui prennent en charge ces patients. Ces deux derniers facteurs interviennent dans l'amélioration de la qualité de vie des patients et dans l'économie de santé.

Ce livre très bien illustré avec une iconographie riche est le fruit de plusieurs années de travail et de recherche de notre collègue le Dr Georges Ha Van, médecin MPR et podologue, directeur d'enseignement du diplôme universitaire « Le pied diabétique » à Paris-VI. Cet ouvrage, qu'il faut lire absolument, constitue une référence sur les principes de la prise en charge du pied diabétique.

\footnotetext{
S. Bendaya $(\triangle)$

Service de médecine physique et de réadaptation fonctionnelle, hôpital Rothschild, 33, boulevard de Picpus, F-75012 Paris, France

e-mail : samy.bendaya@rth.aphp.fr
} 